\title{
Pharmacokinetics of Intranasally Administered Dexmedetomidine in Chinese Children
}

OPEN ACCESS

Edited by: Jun Shi,

Independent Researcher, Shanghai, China

Reviewed by: Rong Zhao,

GenFleet Therapeutics, China Meina Tao Tang, Genentech Inc., United States

${ }^{*}$ Correspondence: Qing-Quan Lian Lianqingquanmz@163.com Hua-Cheng Liu

huachengliu@163.com

tThese authors have contributed equally to this work.

Specialty section:

This article was submitted to Obstetric and Pediatric Pharmacology, a section of the journal Frontiers in Pharmacology

Received: 04 April 2019 Accepted: 11 June 2019 Published: 05 July 2019

Citation:

Wang C-Y, Ihmsen H, Hu Z-Y, Chen J, YeX-F, Chen F, Lu Y, Schüttler J, Lian Q-Q and Liu H-C (2019) Pharmacokinetics of Intranasally Administered Dexmedetomidine in Chinese Children.

Front. Pharmacol. 10:756. doi: 10.3389/fphar.2019.00756

\begin{abstract}
Cheng-Yu Wang ${ }^{1,2 \dagger}$, Harald Ihmsen ${ }^{3 \dagger}$, Zhi-Yan Hu ${ }^{1,2}$, Jia Chen 1,2, Xue-Fei Ye ${ }^{1,2}$, Fang Chen ${ }^{1,2}$, Yi Lu ${ }^{1,2}$, Jürgen Schüttler ${ }^{3}$, Qing-Quan Lian ${ }^{1,2 *}$ and Hua-Cheng Liu ${ }^{1,2 *}$

1 Department of Anesthesiology, Perioperative and Pain Medicine, The Second Affiliated Hospital and Yuying Children's Hospital of Wenzhou Medical University, Zhejiang, China, ${ }^{2}$ Key Laboratory of Anesthesiology of Zhejiang Province, The Second Affiliated Hospital and Yuying Children's Hospital of Wenzhou Medical University, Zhejiang, China, ${ }^{3}$ Department of Anesthesiology, Friedrich-Alexander-University Erlangen-Nürnberg (FAU), Erlangen, Germany
\end{abstract}

Background: Intranasal application is a comfortable, effective, nearly non-invasive, and easy route of administration in children. To date, there is, however, only one pharmacokinetic study on intranasal dexmedetomidine in pediatric populations and none in Chinese children available. Therefore, this study aimed to characterize the pharmacokinetics of intranasally administered dexmedetomidine in Chinese children.

Methods: Thirteen children aged 4 to 10 years undergoing surgery received $1 \mu \mathrm{g} / \mathrm{kg}$ dexmedetomidine intranasally. Arterial blood samples were drawn at various time points until 180 min after dose. Dexmedetomidine plasma concentrations were measured with high performance liquid chromatography (HPLC) and mass spectrometry. Pharmacokinetic modeling was performed by population analysis using linear compartment models with first-order absorption.

Results: An average peak plasma concentration of $748 \pm 30 \mathrm{pg} / \mathrm{ml}$ was achieved after $49.6 \pm 7.2 \mathrm{~min}$. The pharmacokinetics of dexmedetomidine was best described by a twocompartment model with first-order absorption and an allometric scaling with estimates standardized to $70-\mathrm{kg}$ body weight. The population estimates (SE) per $70 \mathrm{~kg}$ bodyweight of the apparent pharmacokinetic parameters were clearance CL/F $=0.32(0.02) \mathrm{L} / \mathrm{min}$, central volume of distribution $\mathrm{V} 1 / \mathrm{F}=34.2(4.9) \mathrm{L}$, intercompartmental clearance $\mathrm{Q} 2 / \mathrm{F}=$ 10.0 (2.2) L/min, and peripheral volume of distribution $\mathrm{V} 2 / \mathrm{F}=34.9$ (2.3) L. The estimated absorption rate constant was $\mathrm{Ka}=0.038(0.004) \mathrm{min}^{-1}$.

Conclusions: When compared with studies in Caucasians, Chinese children showed a similar time to peak plasma concentration after intranasal administration, but the achieved plasma concentrations were about three times higher. Possible reasons are differences in age, ethnicity, and mode of administration.

Keywords: dexmedetomidine, intranasal, pharmacokinetics, pediatrics, Chinese 


\section{INTRODUCTION}

Dexmedetomidine is a highly selective alpha2-adrenergic receptor agonist, which possesses sedative, anxiolytic, and analgesic properties (Belleville et al., 1992). Dexmedetomidine is characterized by a rapid and wide distribution throughout the body and a high-protein binding of about $94 \%$, mainly to albumin and a1-glycoprotein (Weerink et al., 2017). Dexmedetomidine is eliminated mainly through biotransformation by the liver via direct $\mathrm{N}$-glucuronidation by uridine 5 -diphospho-glucuron osyltransferase (UGT2B10, UGT1A4) and via hydroxylation mediated by cytochrome P450 enzymes, mainly CYP2A6 (Weerink et al., 2017). Although dexmedetomidine causes hypertension and bradycardia (Ebert et al., 2000), it has been shown that it can help to provide hemodynamic stability during stressful events in surgery (Kunisawa et al., 2011) and during induction of anesthesia (Yildiz et al., 2006). Furthermore, it has minimal effects on respiration and cognitive function (Belleville et al., 1992; Hsu et al., 2004). In pediatric anesthesia, dexmedetomidine is widely used and plays a significant role as an anesthetic adjuvant, in reducing postoperative pain, emergence agitation, and shivering (Mason and Lerman, 2011). Intranasal application is a nearly noninvasive and easy route of administration, which is comfortable and effective and does not require children's cooperation (Jun et al., 2017). Intranasal administration of dexmedetomidine showed rapid absorption, rapid onset, and high bioavailability (Miller et al., 2018). To decrease side effects and optimize clinical dosing, it is important to get pharmacokinetic information on dexmedetomidine in pediatric patients. The pharmacokinetics of intravenous dexmedetomidine in pediatric patients has been studied in several studies, including premature infants (Potts et al., 2009; Su et al., 2016; Liu et al., 2017). However, although only one pharmacokinetic study of intranasal administered dexmedetomidine has been reported in children in cardiac surgery (Miller et al., 2018), no such data exist in Chinese children. Therefore, the aim of this study was to characterize the pharmacokinetics of intranasal administered dexmedetomidine in Chinese children. Based on previous pharmacokinetic data in adult and pediatric populations (Iirola et al., 2011; Li et al., 2018; Miller et al., 2018), we chose a dose of $1 \mu \mathrm{g} / \mathrm{kg}$, expecting that this should provide adequate sedation and analgesia in the early postoperative phase.

\section{MATERIALS AND METHODS}

This prospective study in a single medical central was conducted in accordance with the guidelines for Good Clinical Practice and the Declaration of Helsinki. The study was approved by the Ethics Committee of the Second Affiliated Hospital of Wenzhou Medical University, China (Reference No. 2017-62) on July 25, 2017. The trial was registered at clinicaltrials.gov on November 9, 2017 (NCT03337581).

\section{Patients}

The enrolment period was from October 20, 2017, to October 21, 2018. As this was not a confirmatory but an exploratory study, sample size estimation was not performed. Sixteen pediatric patients, aged 3 to 10 years, who were scheduled to undergo orthopedics, lower abdominal, urologic, or plastic surgery, were eligible for this study. The expected operation time was at least $2 \mathrm{~h}$. Written informed consent was obtained from the parents or a legal guardian. Exclusion criteria included patients with a history of head trauma history, dexmedetomidine allergy, abnormal liver and kidney function, cardiac conduction system disease, current treatment with digoxin, alpha-adrenergic, or beta-adrenergic agonists or antagonists, clonidine, anti-arrhythmic medications, anticonvulsants, or presence of life-threatening medical conditions. Patients with acute nasal or respiratory symptoms on the day of the study were excluded because of potential interference with intranasal absorption.

\section{Clinical Protocol}

Due to the duration of blood sampling, all participants were scheduled for anesthetization at 8:30 AM. For safety, the patients were fasted from 2:30 AM on the study day until $3 \mathrm{~h}$ after study drug administration. Intake of clear fluids was allowed until 5:30 AM on the study day. There was no premedication used. Heart rate, non-invasive arterial blood pressure, peripheral arterial oxygen saturation, and ECG were monitored continuously. Anesthesia was induced with intravenous administration of fentanyl, $2 \mu \mathrm{g} / \mathrm{kg}$ and propofol, 2 to $3 \mathrm{mg} / \mathrm{kg}$. Endotracheal intubation was facilitated with rocuronium 0.6 to $1.0 \mathrm{mg} / \mathrm{kg}$ before radial arterial cannulation. Mechanical ventilation was carried out with a mixture of 50\% nitrous oxide and 50\% oxygen. During surgical procedure, anesthesia was maintained with sevoflurane 2 to $3 \mathrm{vol} \%$. Repetitive bolus doses of fentanyl $1 \mu \mathrm{g} / \mathrm{kg}$ were given if necessary. The attending anesthesiologist conducted the anesthesia to maintain heart rate and mean arterial blood pressure within $20 \%$ of baseline values.

\section{Dexmedetomidine Dosing}

A dose of $1 \mu \mathrm{g} / \mathrm{kg}$ preservative-free dexmedetomidine $(100 \mu \mathrm{g} / \mathrm{ml}$, Jiangsu Hengrui Pharma Corporation, China) was prepared in a $1-\mathrm{ml}$ syringe. Dexmedetomidine was not diluted and was stored at room temperature for $20 \mathrm{~min}$ to avoid irritating the nasal cavity of children. Thirty minutes before the end of surgery, the patient's head was tilted back to fully expose the nasal cavity. The 1-ml syringe was used to enter the nasal cavity for about $0.5 \mathrm{~cm}$, and the dose of $1 \mu \mathrm{g} / \mathrm{kg}$ dexmedetomidine was administered. After the administration, the nose was squeezed softly to promote the absorption of the drug solution, and the original body position was maintained for $2 \mathrm{~min}$ to prevent the drug solution flowing into the oral cavity through the nasal cavity.

\section{Blood Sampling and Drug Assay}

Arterial blood samples of $0.5 \mathrm{ml}$ each were collected at the following times: immediately before and $3,5,10,15,20$, $30,45,60,90,120,150$, and $180 \mathrm{~min}$ after the intranasal administration. Due to clinical circumstances, longer arterial blood sampling was not feasible. Blood samples were preserved into ethylenediaminetetraacetic acid (EDTA) tubes and stored 
at $4^{\circ} \mathrm{C}$. All heparinized blood samples were centrifuged within 30 min after collection, and the supernatant plasma was pipetted into glass vials and stored at $-20^{\circ} \mathrm{C}$ immediately after separation. Later on, the samples were stored at $-80^{\circ} \mathrm{C}$ until analysis.

Sample preparation was performed by liquid-liquid extraction. Plasma aliquots of $90 \mu \mathrm{l}$ were mixed with $10 \mu \mathrm{l}$ of internal standard solution and extracted with ethyl acetate $(500 \mu \mathrm{l})$. After loading, the sample were vortexed about $2 \mathrm{~min}$. Then, the sample was centrifuged at 15,000 rpm for $10 \mathrm{~min}$, and the supernatant (450 $\mu \mathrm{l}$ ) was transferred into 1.5-ml EDTA tubes. Extracts were evaporated to dryness with a gentle stream of nitrogen, and the dry residue was dissolved in $50-\mu \mathrm{l}$ water. Finally, the sample was centrifuged at $15,000 \mathrm{rpm}$ for $10 \mathrm{~min}$, and the supernatant $(40 \mu \mathrm{l})$ was transferred into EDTA tubes.

Isocratic ultraperformance liquid chromatography was performed with an ACQUITY UPLC BEH C18 1.7- $\mu \mathrm{m} 2.1 \mathrm{~mm} \times$ $50 \mathrm{~mm}$ column (UPLC, Waters Corp., Milford, MA) and a mobile phase consisting of acetonitrile and $0.1 \%$ formic acid in water (28:72; flow rate $0.3 \mathrm{ml} / \mathrm{min}$ ) at $37^{\circ} \mathrm{C}$. A gradient program was used with the mobile phase combining solvent A $(0.1 \%$ formic acid in water) and solvent B (acetonitrile) as follows: 0.0 to $0.5 \mathrm{~min}$ at $28 \%$ $\mathrm{B}, 0.5$ to $1.5 \mathrm{~min}$ linear increase to $90 \% \mathrm{~B}, 1.5$ to $2.0 \mathrm{~min}$ at $90 \% \mathrm{~B}$, and 2.0 to $2.1 \mathrm{~min}$ at $28 \% \mathrm{~B}$. A subsequent re-equilibration time was followed by $1 \mathrm{~min}$ of starting gradient condition. The flow rate was $0.30 \mathrm{ml} / \mathrm{min}$, and the injection volume was $5 \mu \mathrm{l}$. The column and sample temperatures were maintained at $25^{\circ} \mathrm{C}$ and $4^{\circ} \mathrm{C}$, respectively.

Mass spectrometric detection was carried out with an Applied Biosystems API 550 triple-quadrupole instrument, using positive ion spray ionization and multiple reaction monitoring. The needle potential was set to $1.3 \mathrm{kV}$, the source voltages cone was set to $35 \mathrm{~V}$, the temperature of the heated purified air was $300^{\circ} \mathrm{C}$, and the desolvation gas flow was set to $600 \mathrm{~L} / \mathrm{h}$. The collision energy was set to $20 \mathrm{~V}$. The precursor ion-fragment ion pairs detected were $\mathrm{m} / \mathrm{z}$ 200.99-94.98 for dexmedetomidine and $\mathrm{m} / \mathrm{z}$ 205.039-99.014 for the internal standard d4-dexmedetomidine. Quantitation was based on peak area ratios of the analyte and the internal standard.

The assay was linear over a concentration range from 0.01 to $10.0 \mathrm{ng} / \mathrm{ml}$ with a correlation coefficient of 0.999 . The within-run coefficient of variation was $12.2 \%$ at $0.01 \mathrm{ng} / \mathrm{ml}, 11.5 \%$ at 0.20 $\mathrm{ng} / \mathrm{ml}, 4.75 \%$ at $5 \mathrm{ng} / \mathrm{ml}$, and $0.58 \%$ at $10 \mathrm{ng} / \mathrm{ml}$. The betweenbatch coefficient of variation was $1.34 \%$ at $0.20 \mathrm{ng} / \mathrm{ml}, 6.21 \%$ at $5 \mathrm{ng} / \mathrm{ml}$, and $1 \%$ to $2 \%$ at 0.01 and $10 \mathrm{ng} / \mathrm{ml}$. Mean recoveries of dexmedetomidine were better than $82.1 \%$. The recovery of the internal standard $(10 \mathrm{ng} / \mathrm{ml})$ was $89.1 \pm 4.6 \%$. The matrix effect in children plasma was $83.5 \%$ to $103.2 \%$ for dexmedetomidine at different QC levels. The matrix effect for internal standard $(10 \mathrm{ng} / \mathrm{ml}$ ) was $92.2 \pm 6.2 \%$. No apparent matrix effect was found to affect the determination of dexmedetomidine and internal standard in children plasma.

\section{Pharmacokinetic Modeling}

One-, two-, and three-compartment models with first-order absorption were evaluated for pharmacokinetic modeling of dexmedetomidine. The models were parameterized using apparent values of elimination clearance (CL/F), intercompartmental clearances $\left(\mathrm{Q}_{2,3} / \mathrm{F}\right)$, central volume of distribution $\left(\mathrm{V}_{1} / \mathrm{F}\right)$, peripheral volumes of distribution $\left(\mathrm{V}_{2,3} / \mathrm{F}\right)$, and the first-order absorption rate $\left(\mathrm{K}_{\mathrm{a}}\right)$. The analysis was performed with NONMEM (version 7.4.3, ICON Development Solutions), using the first-order conditional estimation method with interaction.

The model parameters were assumed to follow log-normal distributions and the interindividual variability for each structural parameter was modeled as:

$P_{i}=P_{\mathrm{TV}} \cdot \exp \left(\eta_{i}\right)$ where $\mathrm{P}_{\mathrm{i}}$ represents the parameter for the $i$ th individual, $\mathrm{P}_{\mathrm{TV}}$ is the typical value of the parameter in the population, and $\eta_{i}$ is a random variable with zero mean and variance of $\omega^{2}$.

Residual variability was modeled using a combined proportional and additive error: $C m_{i j}=C p_{i j}+C p_{i j} \cdot \varepsilon_{\text {prop }, i j}+\varepsilon_{\text {add }, i j}$ where $\mathrm{Cm}_{i j}$ is the $j$ th observation of the $i$ th subject, $\mathrm{Cp}_{i j}$ is the corresponding predicted value, and $\varepsilon_{\text {prop }, i j}$ and $\varepsilon_{\text {add }, i j}$ are random variables with mean of zero and variance of $\sigma_{\text {prop }}^{2}$ and $\sigma_{\text {add }}^{2}$, respectively.

Model selection was based on the objective function value (OFV) using likelihood ratio tests and on residual analysis. A decrease in the OFV $>6.63\left(P=0.01, \chi^{2}\right.$ distribution with one degree of freedom) was considered statistically significant.

Covariate analysis was conducted in two steps after the base model was identified. First, scatter plots of selected parameter estimates from the basic model versus potential covariates were plotted to explore the covariate-parameter relationships. In the second step, based on the identified potential covariates in step 1, covariate relationships with a linear or a power function were assessed for continuous covariates. The influence of body weight (BW) was modeled as $\mathrm{P}_{i}=\mathrm{P}_{S T D} \cdot\left(\frac{B W i}{70}\right)^{P B W}$ where $\mathrm{P}_{\mathrm{STD}}$ is the standardized value of the parameter for a subject of $70 \mathrm{~kg}$ BW. For the power coefficient PBW, we tested a linear proportional model with $\mathrm{PBW}=1$ for all parameters, an allometric power model with $\mathrm{PBW}=1$ for volumes, and $\mathrm{PBW}=0.75$ for clearances, and a model where the values of PBW were estimated. Further potential covariates included sex, age, serum creatinine $(\mathrm{Cr})$, the ratio of blood urea nitrogen to serum $\mathrm{Cr}$ (BUN/Cr), and $\mathrm{Cr}$ clearance $(\mathrm{CrCL})$, which was calculated using the CockcroftGault formula (Gault et al., 1992).

A stepwise approach was used to evaluate covariate effects. The selecting criteria for a covariate to be included in the model were based on a decrease of $>3.84$ in the OFV $\left(P=0.05, \chi^{2}\right.$ distribution with one degree of freedom) in the forward adding step and an increase of greater than 6.63 in the OFV $\left(P=0.01, \chi^{2}\right.$ distribution with one degree of freedom) in the backward deleting step.

Goodness of fit was assessed graphically and numerically. Model diagnostic graphs included: measured concentrations vs. population predictions and vs. individual predictions; ratio of measured to predicted concentration versus time; conditional weighted residuals versus time and versus population predictions.

Prediction errors were determined for individual and population predictions, and goodness of fit was assessed by 
the median prediction error (MDPE) and the median absolute prediction error (MDAPE) as follows:

$$
\begin{aligned}
\mathrm{MDPE} & =\operatorname{median}\left(\frac{c m_{i j}-c p_{i j}}{c p_{i j}} \times 100 \%\right) \\
\mathrm{MDAPE} & =\operatorname{median}\left(\frac{c m_{i j}-c p_{i j}}{c p_{i j}} \times 100 \%\right)
\end{aligned}
$$

where $\mathrm{Cm}_{\mathrm{ij}}$ and $\mathrm{Cp}_{\mathrm{ij}}$ are the $i$ th measured and predicted concentration of the $j$ th individual, respectively.

A bootstrap analysis with 1,000 replicates (random resampling per subject, with replacement) was performed to analyze the stability of the model parameter estimates and to obtain nonparametric confidence intervals of the model parameters.

Prediction-corrected visual predictive check was used to graphically assess the appropriateness of the pharmacokinetic model (Bergstrand et al., 2011). For this purpose, the concentration profiles were simulated 1,000 times and compared with the observed data to evaluate the predictive performance of the model.

From the individual Bayesian estimates of the model parameters, the following pharmacokinetic variables were also determined: fast distribution half-life $\left(\mathrm{T}^{1} 12 \alpha\right)$, terminal elimination half-life $\left(\mathrm{T}^{1 / 2} \beta\right)$, and time to maximum plasma concentration after intranasal administration (Tpeak). The influence of BW was elucidated by simulating the pharmacokinetics for subjects of different BW.

\section{Statistics}

Data are reported as mean \pm standard deviation (SD) or median (range) unless stated otherwise. All model parameters are reported as estimated values with associated relative standard errors (RSE). Statistical analysis and simulations were performed using Matlab $^{\circledR}$ R2015b (MathWorks, Natick, MA) and R (version 3.5.1).

\section{RESULTS}

\section{Subject Characteristics}

We totally recruited 16 patients. One patient withdrew during the trial and two patients were excluded due to blocked arterial catheterization. In five patients, it was not possible to collect all scheduled blood samples due to clinical circumstances. The last sample was drawn at $150 \mathrm{~min}$ in three subjects, at $120 \mathrm{~min}$ in one subject, and at $60 \mathrm{~min}$ in another subject. In the remaining eight subjects, the last sample was drawn at $180 \mathrm{~min}$ as scheduled. Therefore, the pharmacokinetic analysis was based on 145 samples from 13 children. Demographic and baseline clinical data are summarized in Table $\mathbf{1 .}$

\section{Pharmacokinetic Modeling}

The individual time courses of the dexmedetomidine plasma concentrations are shown in Figure 1. There were no
TABLE 1 | Demographic and baseline clinical data, mean \pm SD (range) or number.

\begin{tabular}{lc}
\hline Characteristics & Values \\
\hline Sex (female/male) & $8 / 5$ \\
Age (years) & $6.5 \pm 1.7(4-10)$ \\
Weight $(\mathrm{kg})$ & $24.8 \pm 5.8(14-36)$ \\
Height $(\mathrm{cm})$ & $110 \pm 12(83-130)$ \\
Body mass index $\left(\mathrm{kg} / \mathrm{m}^{2}\right)$ & $20.2 \pm 1.0(18.5-21.9)$ \\
$\mathrm{Cr}(\mu \mathrm{mol} / \mathrm{L})$ & $37.3 \pm 7.3(23.8-47.0)$ \\
$\mathrm{BUN} / \mathrm{Cr}$ & $0.11 \pm 0.03(0.04-0.15)$ \\
$\mathrm{CrCL}(\mathrm{mL} / \mathrm{min})$ & $99.2 \pm 19.0(78.2-150)$
\end{tabular}

$\mathrm{Cr}$, serum creatinine; BUN/CR, ratio of blood urea nitrogen and creatinine; $\mathrm{CrCL}$, creatinine clearance.

concentrations below the limit of quantitation. The maximum measured dexmedetomidine plasma concentration was $748 \pm$ $29.6(713-795) \mathrm{pg} / \mathrm{ml}$, and the observed time to maximum concentration was $49.6 \pm 7.21(45.0-60.0) \mathrm{min}$.

A linear two-compartment model with first-order absorption $(\mathrm{OFV}=1331.0)$ was significantly better than a one-compartment model $(\mathrm{OFV}=1344.6)$, whereas a three-compartment model could not be estimated reliably. An additional lag time for first-order absorption did not further improve the fit (OFV = 1330.5). Therefore, a two-compartment model with firstorder absorption was chosen as basic structural model. As the estimate of the proportional residual error $\sigma_{\text {prop }}^{2}$ was < 0.0001 , this error term was removed from the model, keeping only the additive residual error $\sigma_{\text {add }}^{2}$. For the basic model, the $\varepsilon$ shrinkage was $13.0 \%$, and the $\eta$ shrinkage of $\mathrm{Ka}, \mathrm{CL} / \mathrm{F}, \mathrm{V}_{1} / \mathrm{F}$, $\mathrm{Q}_{2} / \mathrm{F}$ and $\mathrm{V}_{2} / \mathrm{F}$ was $34.5 \%, 7.05 \%, 26.5 \%, 36.2 \%$, and $18.1 \%$, respectively. Scatter plots of the individual Bayesian parameters indicated an increase of $\mathrm{CL} / \mathrm{F}, \mathrm{V}_{1} / \mathrm{F}$, and $\mathrm{V}_{2} / \mathrm{F}$ with weight. The influence of weight was best described by the allometric power model $(\mathrm{OFV}=1287.1)$ with an exponent of 1 for all volumes and of 0.75 for all clearances. The linear weight model with an exponent of 1 for all parameters showed a worse fit $(\mathrm{OFV}=$ 1288.1). Estimation of the power exponents did not improve

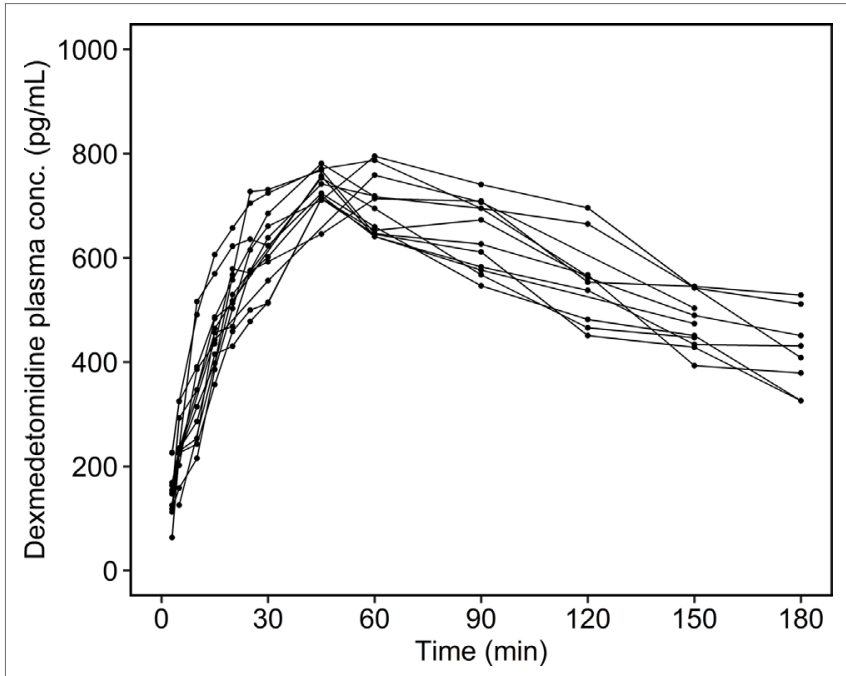

FIGURE 1 | Measured dexmedetomidine plasma concentrations. Each line shows the data of one patient. 
the model $(\mathrm{OFV}=1286.6$ with four additional parameters to be estimated). The interindividual variances of $\mathrm{Ka}, \mathrm{CL} / \mathrm{F}, \mathrm{V}_{1} / \mathrm{F}$, $\mathrm{Q}_{2} / \mathrm{F}$, and $\mathrm{V}_{2} / \mathrm{F}$ changed from $17.3 \%, 27.0 \%, 28.9 \%, 112 \%$, and $40.7 \%$ without weight effect to $10.6 \%, 14.5 \%, 7.55 \%, 162 \%$, and $0.316 \%$ with allometric weight scaling. After inclusion of BW as covariate, there were no further significant effects of age, sex, and laboratory parameters detected.

The estimated parameters of the final pharmacokinetic model are summarized in Table 2 . The interindividual variance of $\mathrm{V}_{2}$ was $<0.0001$ and was therefore fixed to zero. The fast distribution half-life in the population was $1.48 \pm 1.45(0.294-$ 5.16) $\mathrm{min}$, the terminal elimination half-life was $117 \pm 16.1$ (98.0-149) $\mathrm{min}$, and the simulated time to maximum plasma concentration after intranasal administration was $55.2 \pm 5.96$ (42.3-63.4) $\mathrm{min}$.

Median values and confidence intervals of the bootstrap estimates were in good agreement with the population estimates (Table 2). The goodness of fit plots for the final population pharmacokinetic model revealed a high quality of fit with small prediction errors and homogenously distributed residuals (Figure 2). The visual predictive check demonstrated the sufficiency of the model's predictive power (Figure 3).

Table 3 shows the simulated effects of BW on the pharmacokinetics of dexmedetomidine after intranasal administration. Due to the allometric weight scaling, the halflives are not constant but increase with BW. With increasing $\mathrm{BW}$, the maximum plasma concentration is expected to occur later and to be higher.

\section{Side Effects}

Dexmedetomidine slightly decreased the blood pressure and heart rate after intranasal administration (Figure 4). When compared with the baseline, a maximum decrease of $5.4 \pm 4.7 \%$ for mean arterial blood pressure and $3.4 \pm 0.9 \%$ for heart rate was observed $20 \mathrm{~min}$ after administration of dexmedetomidine. The administered dose of $1 \mu \mathrm{g} / \mathrm{kg}$ provided sufficient sedation in the

TABLE 2 | Final population pharmacokinetic parameters.

\begin{tabular}{lcc}
\hline \multicolumn{2}{c}{ Estimate (RSE\%) } & Bootstrap median (95\% Cl) \\
\hline Pharmacokinetic parameters & \\
$\mathrm{Ka}(1 / \mathrm{min})$ & $0.0378(10.2 \%)$ & $0.0379(0.0315-0.0448)$ \\
$\mathrm{CL} / \mathrm{F}(\mathrm{L} / \mathrm{min} / 70 \mathrm{~kg})$ & $0.319(6.6 \%)$ & $0.318(0.283-0.359)$ \\
$\mathrm{V}_{1} / \mathrm{F}(\mathrm{L} / 70 \mathrm{~kg})$ & $34.2(14.4 \%)$ & $33.5(20.3-44.0)$ \\
$\mathrm{Q}_{2} / \mathrm{F}(\mathrm{L} / \mathrm{min} / 70 \mathrm{~kg})$ & $10.0(22.2 \%)$ & $10.6(5.11-34.1)$ \\
$\mathrm{V}_{2} / \mathrm{F}(\mathrm{L} / 70 \mathrm{~kg})$ & $34.9(6.5 \%)$ & $35.3(29.4-46.1)$ \\
Interindividual variability $\omega^{2}$ & & \\
$\mathrm{Ka}$ & $0.0112(50.0 \%)$ & $0.00949(0.00107-0.0203)$ \\
$\mathrm{CL} / \mathrm{F}$ & $0.0207(38.7 \%)$ & $0.0178(0.00452-0.0335)$ \\
$\mathrm{V}_{1} / \mathrm{F}$ & $0.00569(82.7 \%)$ & $0.00516(0.00001-0.0174)$ \\
$\mathrm{Q}_{2} / \mathrm{F}$ & $1.29(50.6 \%)$ & $1.12(0.203-3.18)$ \\
$\mathrm{V}_{2} / \mathrm{F}$ & $0($ fixed $)$ & 0 (fixed) \\
Residual variability & & $1742(1285-2181)$ \\
$\sigma^{2}$ add & $1752(14.6 \%)$ & \\
\hline
\end{tabular}

Ka, absorption rate constant; $C L / F$, apparent clearance; $V_{1} / F$, apparent central volume of distribution; $Q_{2} / F$, apparent intercompartmental clearance; $V_{2} / F$, apparent peripheral volume of distribution; RSE\%, relative standard error; $\mathrm{Cl}$, confidence interval. early postoperative phase. There were no cases of postoperative emergence delirium. The wake-up time after discontinuation of sevoflurane was $20.3 \pm 3.8 \mathrm{~min}$, and the retention time from the postanesthesia care unit was $32.7 \pm 7.1 \mathrm{~min}$.

\section{DISCUSSION}

It was the aim of this study to characterize the pharmacokinetics of intranasal dexmedetomidine in Chinese children. A twocompartment model with first-order absorption and allometric BW scaling was found to describe the data appropriately, whereas age, sex, and laboratory parameters did not show any effect on weight normalized pharmacokinetic parameters.

There are currently only three studies on the pharmacokinetics of intranasal dexmedetomidine available, of which two are in adults (Yoo et al., 2015; Li et al., 2018) and only one in children (Miller et al., 2018). Yoo et al. (2015) administered $84 \mu \mathrm{g}$ (approximately $1 \mu \mathrm{g} / \mathrm{kg}$ ) nasal dexmedetomidine to six healthy adults and found that the peak arterial plasma concentration was 340 (230-700) pg/ $\mathrm{ml}$, and the peak time was 38 (15-60) min. Li et al. (2018) reported for adults a median peak concentration of 250 and $280 \mathrm{pg} / \mathrm{ml}$ after $1 \mu \mathrm{g} / \mathrm{kg}$ dexmedetomidine administered by an atomizer or as drops, respectively. In children aged 6 to 48 months, Miller et al. (2018) found a peak concentration of $182(163-251) \mathrm{pg} / \mathrm{ml}$ and a peak time of 47 (31-62) min after $1 \mu \mathrm{g} / \mathrm{kg}$ dexmedetomidine. The peak time of 45 to $60 \mathrm{~min}$ in the present study is in good agreement with Miller's findings, whereas the shorter peak time in the study by Yoo et al. might have been caused not only by the different age but also by the usage of a higher concentrated dexmedetomidine formulation $(500 \mu \mathrm{g} / \mathrm{ml}$ vs. $100 \mu \mathrm{g} / \mathrm{ml})$. However, the peak concentration after $1 \mu \mathrm{g} / \mathrm{kg}$ dexmedetomidine in the present study was 713 to $795 \mathrm{pg} / \mathrm{ml}$, which is about three times higher than that in the other studies on intranasal dexmedetomidine. Concerning the study by Miller, one has, however, to consider the younger age in that study (6-48 months vs. 4-10 years in the present study). There may be also differences in bioavailability, which has been reported to vary between $41 \%$ (Li et al., 2018) and $84 \%$ (Miller et al., 2018). Finally, dexmedetomidine was applied as drops in the present study, whereas it was administered by an atomizer in the study by Miller et al. (2018). Although Li et al. (2018) did not find differences between application by drops or by atomizer in adults, one cannot rule out such an influence of the intranasal administration in young children. The circumstance that the head was tilted back with the children in a supine position might also have influenced the absorption of dexmedetomidine in the present study. Another possible reason for the observed differences might be alterations in pharmacokinetics due to ethnicity, as the present study was performed in Chinese children whereas Miller et al. and Yoo et al. studied Caucasians. Li et al. found that maximum plasma concentrations after $1 \mu \mathrm{g} / \mathrm{kg}$ in healthy Chinese adults were similar as those reported for Caucasian children and adults in studies by Miller and Yoo. However, in a previous study with intravenous administration of dexmedetomidine, 

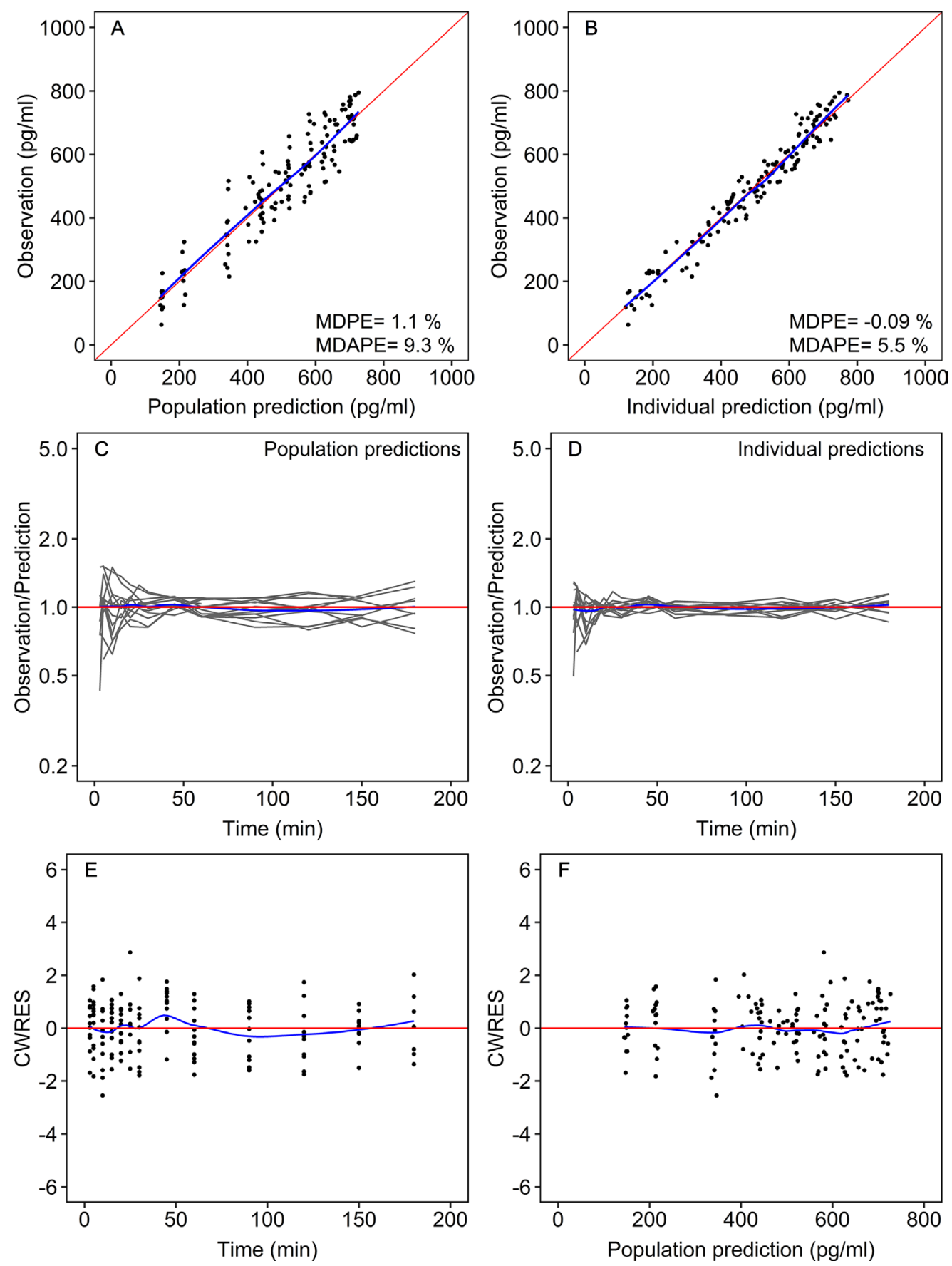

FIGURE 2 | Goodness-of-fit plots for the final population model. Measured dexmedetomidine concentrations versus the population predictions (A) and versus the individual Bayesian predictions (B). Ratio of the measured to predicted concentrations vs. time for the population predictions (C) and the individual predictions (D). Conditional weighted residuals (CWRES) versus time (E) and versus population predicted plasma dexmedetomidine concentrations (F). Each gray line shows the data of one patient. The red lines are lines of identity (measured = predicted). The blue lines are smoothers through the data. MDPE, median prediction error; MDAPE, median absolute prediction error.

Liu et al. (2017) found that Chinese children showed a similar clearance, but larger volumes of distribution when compared with studies in Caucasians. Altogether, such pharmacokinetic alterations due to ethnicity alone may not fully explain the higher concentrations observed in the present study.
Corresponding to the higher observed concentrations, the present estimates of clearance and volume of distribution were lower than in previous studies. Whereas the apparent elimination clearance was $0.32 \mathrm{~L} / \mathrm{min}$ per $70 \mathrm{~kg}$ in the present study, Miller et al. found a systemic clearance of $1.04 \mathrm{~L} / \mathrm{min}$ per $70 \mathrm{~kg}$ with a bioavailability 


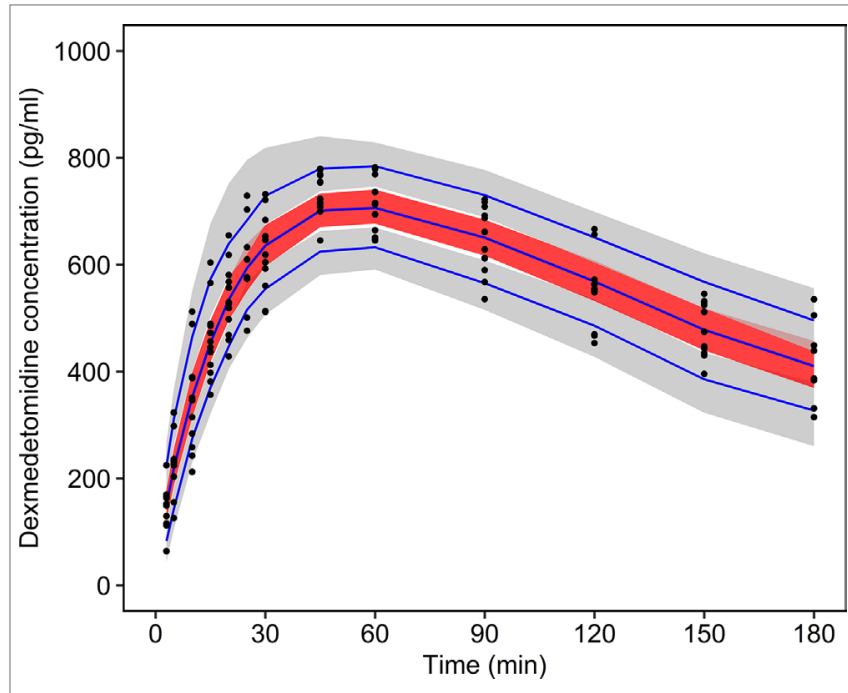

FIGURE 3 | Prediction-corrected visual predictive check. The measured dexmedetomidine concentrations are plotted as black dots. The blue lines show the 5\%,50\%, and 95\% quantiles of the predictions. The gray shaded areas show the $90 \%$ confidence intervals of the $5 \%$ and $95 \%$ prediction quantiles. The red shaded area shows the $90 \%$ confidence interval of the $50 \%$ prediction quantile.

TABLE 3 | Pharmacokinetic parameters for a child of 10, 20, 30, and $40 \mathrm{~kg}$ body weight, as obtained from the final pharmacokinetic model.

\begin{tabular}{lcccc}
\hline & $\mathbf{1 0} \mathbf{~ k g}$ & $\mathbf{2 0} \mathbf{~ k g}$ & $\mathbf{3 0 ~} \mathbf{k g}$ & $\mathbf{4 0} \mathbf{~ k g}$ \\
\hline $\mathrm{CL} / \mathrm{F}(\mathrm{L} / \mathrm{min})$ & 0.0740 & 0.124 & 0.169 & 0.209 \\
$\mathrm{~V}_{1} / \mathrm{F}(\mathrm{L})$ & 4.88 & 9.76 & 14.6 & 19.5 \\
$\mathrm{~V}_{\mathrm{SS}} / \mathrm{F}(\mathrm{L})$ & 9.87 & 19.7 & 29.6 & 39.5 \\
$\mathrm{~T} 1 / 2 \boldsymbol{\alpha}(\mathrm{min})$ & 0.73 & 0.87 & 0.96 & 1.03 \\
$\mathrm{~T} 1 / 2 \beta(\mathrm{min})$ & 93.1 & 111 & 123 & 132 \\
$\mathrm{~T}_{\text {peak }}(\mathrm{min})$ & 52.4 & 55.6 & 57.5 & 58.9 \\
$\mathrm{C}_{\text {peak }}(\mathrm{pg} / \mathrm{ml})$ & 675 & 704 & 720 & 731 \\
\hline
\end{tabular}

$C L / F$, apparent clearance; $V_{1} / F$, apparent central volume of distribution; $V_{S S} / F$, apparent volume of distribution at steady-state; $T 1 / 2 \alpha$, fast distribution half-life; $T 1 / 2 \beta$, terminal elimination half-life; $T_{\text {peak }}$, time to maximum plasma concentration after intranasal bolus administration; $C_{\text {peaks }}$ maximum plasma concentration after an intranasal bolus dose of $1 \mu \mathrm{g} / \mathrm{kg}$.

of $84 \%$. The apparent central volume of distribution in the present study was $34.2 \mathrm{~L} / 70 \mathrm{~kg}$ compared with $59.8 \mathrm{~L} / 70 \mathrm{~kg}$ in the study by Miller. However, one has to consider the differences in age and in ethnicity between the two studies. Furthermore, Miller et al. studied cardiac patients, whereas we studied children undergoing orthopedics, lower abdominal, urologic, or plastic surgery. In a previous study in Chinese children aged 1 to 9 years, Liu et al. (2017) found a systemic clearance of $0.60 \mathrm{~L} / \mathrm{min} / 70 \mathrm{~kg}$ and a central volume of distribution of $84.3 \mathrm{~L} / 70 \mathrm{~kg}$ after short intravenous infusion, which is also higher than in the present study. However, one has to consider that venous blood samples were analyzed in that study, whereas arterial concentrations were used in the present study.

Concerning the effect of covariates on dexmedetomidine pharmacokinetics, previous studies found a significant influence of BW (Potts et al., 2009; Liu et al., 2017; Miller et al., 2018). In the present study, BW was also found to be a significant covariate, and

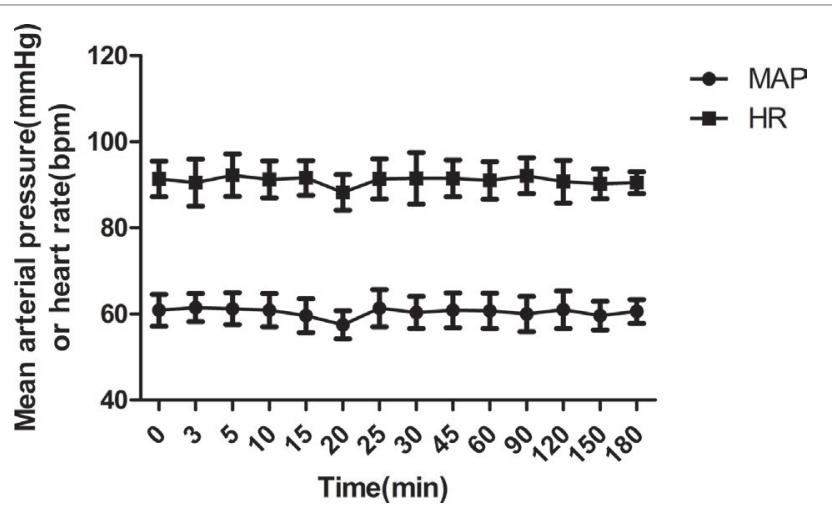

FIGURE 4 | Mean arterial blood pressure (MAP) and heart rate (HR) during the study period (mean $\pm \mathrm{SD}$ ).

as in previous studies, an allometric scaling with an exponent of 0.75 for clearances and an exponent of 1.0 for volumes was most appropriate to assess this influence. The estimation of the power exponents did not improve the model. Furthermore, Anderson and Holford (2008) proposed to fix the allometric coefficients because estimates of these parameters may be quite imprecise. A model with a linear proportional weight scaling with an exponent of 1.0 for all parameters was only slightly worse than the allometric model. This may be explained by the relatively narrow range of BW (14-36 kg) in the present study, so that the difference between a power model with an exponent of 0.75 and a linear proportional model is not as pronounced. On the other hand, the allometric scaling of clearance with an exponent of 0.75 is an established and well-supported model to account for the effect of size in pharmacokinetics (Anderson and Holford, 2008). Whereas the interindividual variabilities of the apparent elimination clearance CL/F and the apparent volumes of distribution were clearly reduced after BW scaling, this was not the case for the intercompartmental clearance Q2/F. This means that the large interindividual variability of this parameter remains unexplained. One has, however, to consider that Q2/F was the parameter with the highest shrinkage and the highest RSE. Additionally, the standard error of the interindividual variability for this parameter was also relatively high. Thus, this parameter was not as well estimable as the other parameters. This might be caused by the relative small sample size of 13 subjects and the relatively short sampling time of $180 \mathrm{~min}$. The power model for the weight effect has the consequence that the maximum plasma concentration after intranasal administration should occur later and should be higher for a child with higher BW (Table 3). Furthermore, the recovery might be longer as the terminal half-life also increases with weight. However, these weight effects are only small or moderate.

Age had no effect on dexmedetomidine pharmacokinetics in the present study. This is reasonable if one considers that the age effect on clearance, which was reported by Potts et al. (2009), was most prominent in the younger than 2 years range, whereas the age in our study population was 4 to 10 years. The present finding that renal function as assessed by CrCL had no effect is also in agreement with previous findings (De Wolf et al., 2001).

In previous studies, it was also found that dexmedetomidine clearance was affected by the cardiac output in a way that 
dexmedetomidine reduces cardiac output, which in turn leads to a reduction of dexmedetomidine clearance (Dutta et al., 2000; Iirola et al., 2012; Li et al., 2018). In this study, cardiac output was not monitored. However, the reported cardiac output effect was prominent at dexmedetomidine concentrations greater than $1,000 \mathrm{pg} / \mathrm{ml}$, which were not reached in this study. As the observed changes in blood pressure and heart rate were also small, one may assume no effect of dexmedetomidine on the cardiac output in the present study.

The effect of dexmedetomidine on hemodynamics is mainly dependent on plasma drug concentration and intravenous injection rate, and a biphasic cardiovascular response has been reported for dexmedetomidine with a transient hypertension followed by hypotension (Ebert et al., 2000). The transient hypertension can be avoided by dosing regimens with a slow increase of concentration (Dyck et al., 1993). Dexmedetomidine causes peripheral vasoconstriction and bradycardia when the plasma concentration exceeds $1,000 \mathrm{pg} / \mathrm{ml}$ (Ebert et al., 2000). In the present study, mean arterial pressure and heart rate slightly decreased after $20 \mathrm{~min}$ of administration, but no increase in blood pressure was observed. This may be explained by the slow concentration increase after nasal administration compared with intravenous administration and by the relatively low peak plasma concentrations.

Concerning the therapeutic window of dexmedetomidine for sedation in children, it has been proposed that a range of 200 to $600 \mathrm{pg} / \mathrm{ml}$ may be appropriate for procedural sedation (Miller et al., 2018). The plasma concentrations in the present study were higher, but the sedation in the early postoperative phase was satisfactory without serious side effects. One may therefore conclude that an intranasal dose of $1 \mu \mathrm{g} / \mathrm{kg}$ is appropriate for sedation in the early postoperative phase in Chinese children in the studied range of age and weight.

The present study has several limitations. First, the blood sampling time was only $3 \mathrm{~h}$, whereas a longer sampling time might have allowed the estimation of a three-compartment model. In previous pharmacokinetic studies of intravenous and also intranasal administration of dexmedetomidine in children, however, a twocompartment model was also found to be appropriate, even if longer blood sampling was performed (Potts et al., 2009; Liu et al., 2017; Miller et al., 2018). A sampling time of about three times the terminal half-life (i.e., $6 \mathrm{~h}$ for dexmedetomidine) is generally recommended for pharmacokinetic studies. Due to clinical circumstances it was, however, not feasible to collect arterial blood samples longer than $3 \mathrm{~h}$, and a combination of venous and arterial samples would have also not been appropriate. Furthermore, the

\section{REFERENCES}

Anderson, B. J., and Holford, N. H. (2008). Mechanism-based concepts of size and maturity in pharmacokinetics. Annu. Rev. Pharmacol. Toxicol. 48, 303-332. doi: 10.1146/annurev.pharmtox.48.113006.094708

Belleville, J. P., Ward, D. S., Bloor, B. C., and Maze, M. (1992). Effects of intravenous dexmedetomidine in humans. I. Sedation, ventilation, and metabolic rate. Anesthesiology 77, 1125-1133. doi: 10.1097/00000542-199212000-00013

Bergstrand, M., Hooker, A. C., Wallin, J. E., and Karlsson, M. O. (2011). Predictioncorrected visual predictive checks for diagnosing nonlinear mixed-effects models. AAPS J. 13, 143-151. doi: 10.1208/s12248-011-9255-Z terminal half-life of about $2 \mathrm{~h}$ in the present study is in agreement with the findings of previous studies. In five subjects, the last concentration was drawn before $180 \mathrm{~min}$. The impact on the results, however, was negligible, as the parameter estimates were very similar when these five subjects were removed from the data set. A further limitation is the circumstance that there was no intravenous administration of dexmedetomidine performed, so that the bioavailability after intranasal administration could not be determined. Finally, as the range of age and weight in the study population was relatively small, we cannot draw conclusions about the pharmacokinetics of intranasal administered dexmedetomidine in infants younger than 4 years or in obese children.

In conclusion, the pharmacokinetics of intranasal dexmedetomidine could be well described by a two-compartment model with first-order absorption and allometric BW scaling. When compared with the studies in Caucasians, Chinese children showed a similar time to peak plasma concentration, but the achieved plasma concentrations were about three times higher. The reason for this observation is not completely clear, but differences in age, ethnicity, and mode of administration may be responsible. Therefore, further studies in larger populations would be helpful.

\section{DATA AVAILABILITY}

Publicly available datasets were analyzed in this study. This data can be found here: www.clinicaltrials.gov.on

\section{AUTHOR CONTRIBUTIONS}

$\mathrm{Z}-\mathrm{YH}$ and JC contributed to the drug assay. X-FY, FC and YL contributed to the sample collection. IS contributed to the pharmacokinetic analysis. C-YW and HI contributed to the pharmacokinetic analysis and writing of the article. Q-QL and $\mathrm{H}-\mathrm{CL}$ contributed to the study design.

\section{FUNDING}

The research was funded by Zhejiang provincial natural science foundation (LY17H310006), Clinical Research Foundation of the Second Affiliated Hospital of Wenzhou Medical University (SAHoWMUCR2018-03-126) and Zhejiang provincial public welfare technology application research foundation of China (2015C33100). 
Ebert, T. J., Hall, J. E., Barney, J. A., Uhrich, T. D., and Colinco, M. D. (2000). The effects of increasing plasma concentrations of dexmedetomidine in humans. Anesthesiology 93, 382-394. doi: 10.1097/00000542-200008000-00016

Gault, M. H., Longerich, L. L., Harnett, J. D., and Wesolowski, C. (1992). Predicting glomerular function from adjusted serum creatinine. Nephron 62, 249-256. doi: 10.1159/000187054

Hsu, Y. W., Cortinez, L. I., Robertson, K. M., Keifer, J. C., Sum-Ping, S. T., Moretti, E. W., et al. (2004). Dexmedetomidine pharmacodynamics: part I: crossover comparison of the respiratory effects of dexmedetomidine and remifentanil in healthy volunteers. Anesthesiology 101, 1066-1076. doi: 10.1097/00000542-200411000-00005

Iirola, T., Vilo, S., Manner, T., Aantaa, R., Lahtinen, M., Scheinin, M., et al. (2011). Bioavailability of dexmedetomidine after intranasal administration. Eur. J. Clin. Pharmacol. 67, 825-831. doi: 10.1007/s00228-011-1002-y

Iirola, T., Ihmsen, H., Laitio, R., Kentala, E., Aantaa, R., Kurvinen, J. P., et al. (2012). Population pharmacokinetics of dexmedetomidine during long-term sedation in intensive care patients. Br. J. Anaesth. 108, 460-468. doi: 10.1093/bja/aer441

Jun, J. H., Kim, K. N., Kim, J. Y., and Song, S. M. (2017). The effects of intranasal dexmedetomidine premedication in children: a systematic review and metaanalysis. Can. J. Anaesth. 64, 947-961. doi: 10.1007/s12630-017-0917-x

Kunisawa, T., Ueno, M., Kurosawa, A., Nagashima, M., Hayashi, D., Sasakawa, T., et al. (2011). Dexmedetomidine can stabilize hemodynamics and spare anesthetics before cardiopulmonary bypass. J. Anesth. 25, 818-822. doi: 10.1007/s00540-011-1215-3

Li, A., Yuen, V. M., Goulay-Dufay, S., Sheng, Y., Standing, J. F., Kwok, P. C. L., et al. (2018). Pharmacokinetic and pharmacodynamic study of intranasal and intravenous dexmedetomidine. Br. J. Anaesth. 120, 960-968. doi: 10.1016/j. bja.2017.11.100

Liu, H. C., Lian, Q. Q., Wu, F. F., Wang, C. Y., Sun, W., Zheng, L. D., et al. (2017). Population pharmacokinetics of dexmedetomidine after short intravenous infusion in Chinese children. Eur. J. Drug. Metab. Pharmacokinet. 42, 201-211. doi: 10.1007/s13318-016-0333-6

Mason, K. P., and Lerman, J. (2011). Review article: dexmedetomidine in children: current knowledge and future applications. Anesth. Analg. 113, 1129-1142. doi: 10.1213/ANE.0b013e31822b8629
Miller, J. W., Balyan, R., Dong, M., Mahmoud, M., Lam, J. E., Pratap, J. N., et al. (2018). Does intranasal dexmedetomidine provide adequate plasma concentrations for sedation in children: a pharmacokinetic study. Br. J. Anaesth. 120, 1056-1065. doi: 10.1016/j.bja.2018.01.035

Potts, A. L., Anderson, B. J., Warman, G. R., Lerman, J., Diaz, S. M., and Vilo, S. (2009). Dexmedetomidine pharmacokinetics in pediatric intensive care-a pooled analysis. Paediatr. Anaesth. 19, 1119-1129. doi: 10.1111/j.1460-9592.2009.03133.x

Su, F., Gastonguay, M. R., Nicolson, S. C., DiLiberto, M., Ocampo-Pelland, A., and Zuppa, A. F. (2016). Dexmedetomidine pharmacology in neonates and infants after open heart surgery. Anesth. Analg. 122, 1556-1566. doi: 10.1213/ ANE.0000000000000869

Weerink, M. A. S., Struys, M., Hannivoort, L. N., Barends, C. R. M., Absalom, A. R., and Colin, P. (2017). Clinical pharmacokinetics and pharmacodynamics of dexmedetomidine. Clin. Pharmacokinet. 56, 893-913. doi: 10.1007/ s40262-017-0507-7

Yildiz, M., Tavlan, A., Tuncer, S., Reisli, R., Yosunkaya, A., and Otelcioglu, S. (2006). Effect of dexmedetomidine on haemodynamic responses to laryngoscopy and intubation: perioperative haemodynamics and anaesthetic requirements. Drugs R. D. 7, 43-52. doi: 10.2165/00126839-200607010-00004

Yoo, H., Iirola, T., Vilo, S., Manner, T., Aantaa, R., Lahtinen, M., et al. (2015). Mechanism-based population pharmacokinetic and pharmacodynamic modeling of intravenous and intranasal dexmedetomidine in healthy subjects. Eur. J. Clin. Pharmacol. 71, 1197-1207. doi: 10.1007/s00228-015-1913-0

Conflict of Interest Statement: The authors declare that the research was conducted in the absence of any commercial or financial relationships that could be construed as a potential conflict of interest.

Copyright (c) 2019 Wang, Ihmsen, Hu, Chen, Ye, Chen, Lu, Schüttler, Lian and Liu. This is an open-access article distributed under the terms of the Creative Commons Attribution License (CC BY). The use, distribution or reproduction in other forums is permitted, provided the original author(s) and the copyright owner(s) are credited and that the original publication in this journal is cited, in accordance with accepted academic practice. No use, distribution or reproduction is permitted which does not comply with these terms. 